\title{
INFLUENCE DE LA FERTILISATION SUR LA QUALITÉ DU BOIS DE PIN MARITIME
}

\author{
H. POLGE \\ Station de Recherches sur la Qualité des Bois, \\ Centre national de Recherches forestières, 54 - Nancy \\ Institut national de la Recherche agronomique
}

\section{SOMMAIRE}

Au cours de diverses expériences, a été analysée l'influence de la fertilisation, sur la densité globale, les composantes de la densité, le retrait longitudinal, le rendement en fibres et la longueur des fibres du bois de Pin maritime ; elle est parfois significative, mais toujours faible, et ne saurait, quand elle est défavorable, contrebalancer les gains de croissance très importants qui ont été observés.

\section{1. - INTRODUCTION}

Les techniques de fertilisation, qui commencent à se répandre en sylviculture moderne, permettent souvent d'obtenir des résultats spectaculaires au point de vue production en volume. Tel est le cas notamment de l'expérience française la plus ancienne et la mieux analysée jusqu'ici : celle de Mimizan sur Pin maritime, où Guinaudeau, Illy, Maugé et Dumas (1963) ont pu mettre en évidence une action très favorable des engrais, scories de déphosphoration en particulier, sur la croissance, la régularité des pousses en hauteur, la floraison, la durée de vie des aiguilles et la mycorhization.

Mais toutes ces modifications traduisent en fait autant de changements dans l'équilibre physiologique des arbres et sont donc susceptibles d'avoir des répercussions notables sur la structure anatomique du bois produit et, par suite, sur ses caractéristiques technologiques.

Il était normal que la Station de Recherches sur la Qualité des Bois se préoccupe de savoir s'il en était bien ainsi, si ces conséquences éventuelles étaient favorables ou défa- 
vorables, et d'examiner, dans cette dernière hypothèse, si elles étaient, ou non, susceptibles de contrebalancer les avantages apportés par la fertilisation dans les autres domaines sus-indiqués.

Tel a été le but d'un certain nombre d'études successives, certaines très sommaires, d'autres plus élaborées, dont il est rendu compte ci-dessous.

\section{2. - EXPÉRIENCE DE MIMIZAN. PRÉLÈVEMENTS DE 1964}

\section{1. - MATÉRIEL ET MÉTHODES}

Pour cette première étude, entreprise sur des plants de 7 ans, le matériel d'expérience a consisté en carottes de sondage de $5 \mathrm{~mm}$ de diamètre, extraites à $40 \mathrm{~cm}$ environ au-dessus du niveau du sol, alternativement sur les faces nord et sud des arbres, de façon à éviter le bois anormal lié à l'anisotropie du Pin maritime des Landes qui est, on le sait, maximale dans le sens est-ouest (Polge et ILLY, 1967).

Une première série de prélèvements a été faite de façon systématique à raison d'un plant tous les dix, en suivant les lignes de semis dont l'orientation générale est précisément est-ouest, et l'échantillonnage ainsi obtenu représenterait bien la moyenne des placeaux étudiés si l'on n'avait malheureusement dû éliminer les plants de diamètre inférieur à $3 \mathrm{~cm}$ qui risquaient d'éclater littéralement lors de l'introduction des tarières. Malgré cette restriction, qui n'a pu qu'occasionner une réduction des écarts de toute nature constatés entre les arbres traités et non traités, le diamètre moyen de ces derniers est resté sensiblement plus faible que celui des fertilisés, et il a paru par suite intéressant, au moins pour la plus importante des caractéristiques étudiées : la densité globale, de comparer également les arbres ayant bénéficié d'un apport d'engrais à des témoins de même taille; tel a été l'objet d'un second échantillonnage, volontairement biaisé, constitué par les plus gros témoins.

Les prélèvements ont été exécutés dans les trois premiers blocs; ils ont porté uniquement sur les témoins et les arbres des placeaux avec phosphore seul, puisque c'est cet élément qui a provoqué de façon à peu près exclusive l'accélération constatée de la croissance des jeunes Pins ; le nombre total d'arbres variant d'une parcelle unitaire à l'autre, il en a été de même pour l'échantillonnage au 1/10. Il n'était dès lors pas possible de procéder à une analyse statistique orthogonale, et on s'est contenté, pour ce premier essai, d'établir des tests de comparaison de moyennes portant sur l'ensemble des échantillons de chaque traitement.

\section{2. - RÉSULTATS}

\subsection{Retrait longitudinal}

On sait qu'un retrait longitudinal excessif affecte le bois juvénile des Conifères et est en grande partie responsable de ses médiocres qualités technologiques.

Il était donc normal d'étudier, en premier lieu, l'incidence de la fertilisation sur cette importante caractéristique physique. Les résultats obtenus ont été les suivants :

TABLEAU 1

\begin{tabular}{|c|c|c|c|}
\hline & Témoins & Fertilisés & Test $\langle\boldsymbol{t}\rangle$ \\
\hline Nombre d'échantillons & 116 & 135 & \\
\hline $\begin{array}{l}\text { Retrait longitudinal moyen de l'état saturé } \\
\text { à l'état sec à l'air ( } 8 \% \text { d'humidité) } \ldots . .\end{array}$ & $0,85 \%$ & $0,98 \%$ & $1,36 \mathrm{NS}$ \\
\hline
\end{tabular}


Il y a donc une tendance non significative à l'aggravation du retrait axial dans les Pins fertilisés ; elle s'est surtout manifestée dans le bloc 1 où le retrait moyen est de $0,89 \%$ pour les 48 témoins et $1,30 \%$ pour les 45 arbres du placeau à phosphore avec une ( $t$ ) significatif de 2,29.

\subsection{Densité globale}

La comparaison se présente comme suit :

TABLEAU 2

\begin{tabular}{|c|c|c|c|}
\hline & $\begin{array}{c}\text { Nombre } \\
\text { d'échantillons }\end{array}$ & $\begin{array}{l}\text { Infra-densité } \\
\text { en } \mathrm{g} / \mathrm{dm}^{3}\end{array}$ & Test « $t »$ \\
\hline Témoins ordinaires $\ldots$ & 116 & 357 & \multirow{3}{*}{$\begin{array}{ll}2,41 * \\
& 4,87^{* *}\end{array}$} \\
\hline Fertilisés $\ldots \ldots \ldots \ldots \ldots \ldots \ldots \ldots \ldots$ & 135 & 351 & \\
\hline Témoins biaisés $\ldots \ldots \ldots \ldots \ldots \ldots \ldots$ & 20 & 374 & \\
\hline
\end{tabular}

La baisse de densité du bois occasionnée par la fertilisation est donc faible $(6 \mathrm{~kg}$ de matière sèche par $\mathrm{m}^{3}$ ), mais significative.

Elle apparaît cependant beaucoup plus importante lorsqu'on compare les arbres ayant bénéficié d'un apport de phosphore aux témoins biaisés de diamètre sensiblement égal. Il est probable que cet état de choses résulte de différences génotypiques : les plus gros témoins sont en effet vraisemblablement des arbres qui, dans un environnement donné, sont plus capables que les autres de prolonger leur croissance en diamètre à l'automne, ce qui leur confère ipso facto une aptitude particulière à produire du bois dense ; les arbres fertilisés, au contraire, n'acquièrent des dimensions comparables qu'à la suite d'une modification du milieu qui, favorisant la pousse en hauteur, pourrait, par voie de conséquence, entraîner un apport plus grand d'auxines élaborées au niveau des bourgeons, d'où la production de trachéides à larges lumens et à membranes minces, donc de bois de faible densité.

\subsection{Composantes de la densité}

Après avoir radiographié toutes les carottes de sondage de l'expérience, on a sélectionné celles qui étaient les plus radiales et dont, en outre, les images aux rayons $\mathrm{X}$ étaient les plus nettes ; ce sont là deux critères tout à fait indépendants de la densité du bois, en sorte que l'échantillonnage obtenu peut être considéré comme prélevé au hasard.

Des profils densitométriques ont été dressés pour les 23 arbres fertilisés et les 23 témoins ainsi choisis ; on y a relevé, sur trois accroissements annuels consécutifs, ceux de 1961, 1962 et 1963, les densités maximum et minimum annuelles, les largeurs de cernes, ainsi que celles des fractions de cernes de densités égales ou supérieures à 500,600 et $700 \mathrm{~g} / \mathrm{dm}^{3}$, toutes mesures faites à l'état ( sec à l'air ) (8\% d'humidité environ). 
Des tests ( $t$ )) ont permis d'établir quelles étaient, parmi les diverses caractéristiques, celles qui différaient significativement entre témoins et arbres traités ; les résultats ci-dessous ont été obtenus :

\section{TABLEAU 3}

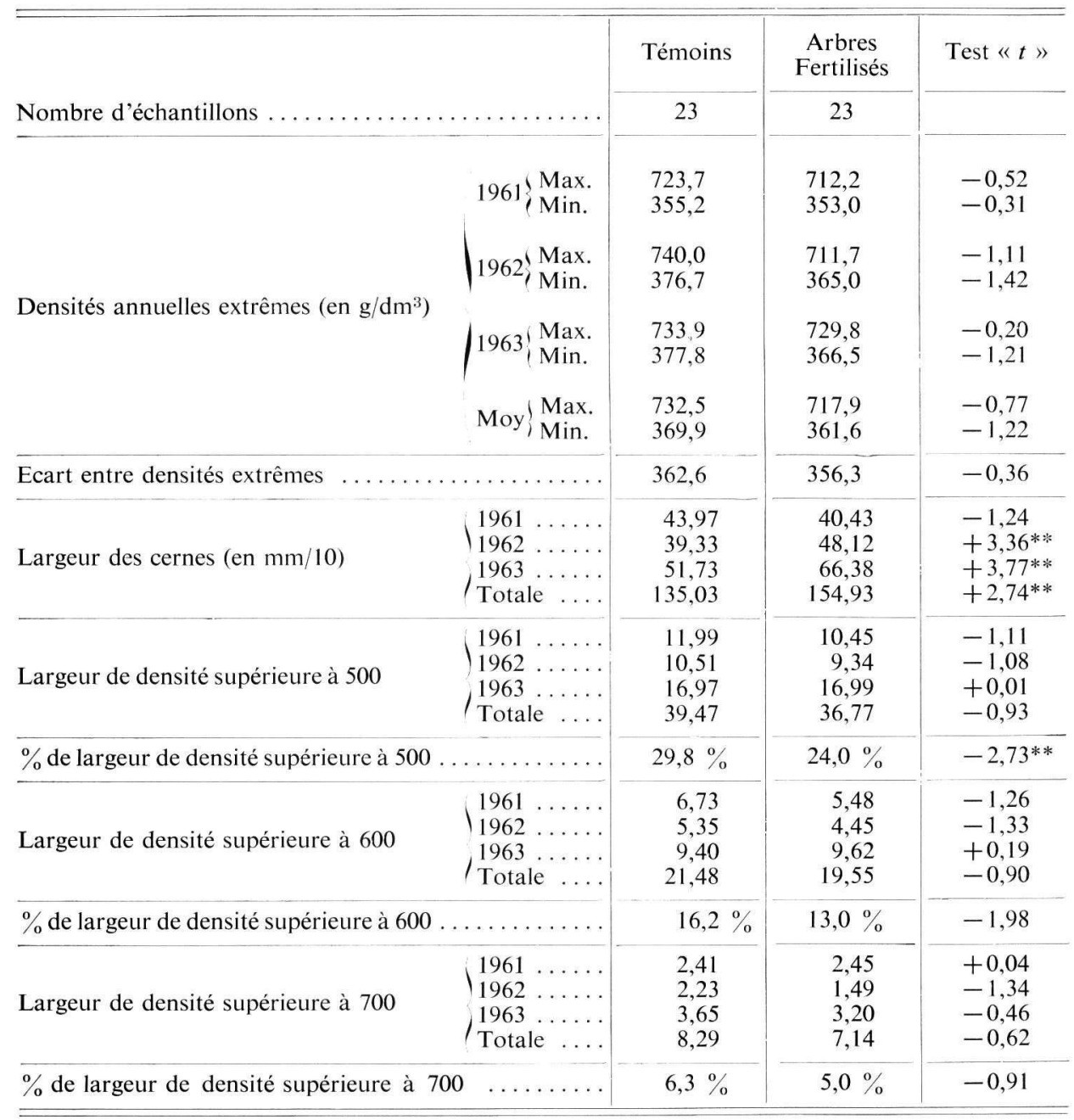

On doit tout d'abord mettre en garde contre une confusion possible entre les diverses valeurs des densités instantanées indiquées ci-dessus et celles des densités globales données précédemment ; elles ne sont pas comparables pour deux raisons : tout d'abord on n'a étudié ici que les trois derniers cernes, alors que les carottes de sondage en comprenaient souvent 4 ou 5 , et les tout premiers accroissements côté moelle ainsi éliminés peuvent très bien avoir une structure sensiblement différente 
des suivants ; en outre, les densités globales sont exprimées en infra-densité (poids anhydre divisé par volume saturé), alors que les densités instantanées sont calculées sur la base du poids et du volume à l'état sec à l'air, et correspondent donc à des rapports dont les numérateurs sont plus forts (à cause de l'eau contenue) et les dénominateurs plus faibles (en raison du retrait du bois).

Cette réserve étant faite, le tableau 3 appelle les commentaires suivants :

- Toutes les valeurs extrêmes des densités annuelles sont moindres pour les arbres fertilisés que pour les témoins ; les différences ne sont certes pas significatives (en raison vraisemblablement d'un échantillonnage trop faible), mais se produisent toutes dans le même sens.

Les densités maximales sont davantage affectées que les densités minimales (baisse de $14,6 \mathrm{~g} / \mathrm{dm}^{3}$ contre 8,3 ) et on observe par suite une certaine tendance à une diminution du contraste de densité à l'intérieur des cernes, donc à une amélioration des caractéristiques technologiques liée à une moindre hétérogénéité.

- L'étude des largeurs de cernes confirme dans l'ensemble ce que l'on savait déjà au sujet de la stimulation de la croissance occasionnée par la fertilisation.

Les gains sont très significatifs pour les accroissements de 1962 et de 1963 , ainsi que pour l'ensemble de la période 1961 à 1963 ; ils se chiffrent approximativement à $1 \mathrm{~mm}$ sur le rayon en 1962, 1,5 mm en 1963 et $2 \mathrm{~mm}$ pour le total des trois années en cause. On doit cependant noter qu'en 1961 la largeur moyenne des accroissements a été sensiblement plus faible dans les arbres fertilisés que dans les témoins, la différence n'étant, il est vrai, pas significative ; cette anomalie paraît difficilement compréhensible, mais toute tentative d'explication devrait sans doute tenir compte du fait que l'année 1961 a été exceptionnellement chaude dans les Landes, surtout en février et en septembre, et aussi anormalement sèche pendant presque toute la période de végétation.

- Alors que les accroissements des témoins sont généralement plus faibles que ceux des arbres ayant bénéficié d'un apport de phosphore, les largeurs des zones de densité égale ou supérieure aux diverses densités de référence sont au contraire le plus souvent plus grandes chez les premiers, d'où résultent des différences très importantes lorsque l'on passe des valeurs absolues aux pourcentages ; sur l'ensemble des trois années étudiées, la diminution est très significative pour la fraction de longueur radiale dont la densité excède $500 \mathrm{~g} / \mathrm{dm}^{3}$, et elle est extrêmement voisine du seuil de signification lorsque la limite est fixée à $600 \mathrm{~g} / \mathrm{dm}^{3}$.

Cette réduction de la proportion de bois dense, qui rend parfaitement compte de la baisse de la densité globale, peut, d'une manière générale, être considérée comme défavorable ; elle laisse présager à la fois une production de fibres (rapportée au volume apparent sur pied) plus faible et une détérioration de toutes les caractéristiques mécaniques, à l'exception de la compression de flanc (KELLER, 1968).

\section{3. - EXPÉRIENCE DE RÉTIS. PRÉLÈVEMENTS DE 1964}

Il s'agit là d'un tout petit essai dont les résultats ne sont donnés ici que parce qu'ils confirment, sur une lande plus humide et nettement plus pauvre, ceux obtenus 
à Mimizan en ce qui concerne la densité globale. Une description sommaire du dispositif et un résumé des résultats obtenus au point de vue croissance en hauteur ont fait l'objet d'une publication par l'AFOCEL (1960-65).

Rappelons que les arbres fertilisés avaient été semés sur des bandes ayant reçu en couverture 300 unités d'acide phosphorique et 300 unités de potasse à l'hectare, et que, au cours des $3^{\mathrm{e}}$ et $4^{\mathrm{e}}$ années après le semis, les interbandes ont été travaillées au rotavator, et ont, à cette occasion, bénéficié d'un apport de 100 unités d'acide phosphorique et de potasse, plus 80 unités d'azote.

Des prélèvements à la tarière, effectués à l'âge de 6 ans sur 67 arbres traités et 23 témoins, ont servi à la détermination des infra-densités moyennes qui ont été les suivantes :

- témoins

- arbres fertilisés

$$
\begin{aligned}
& : 364,7 \mathrm{~g} / \mathrm{dm}^{3} ; \\
& : 349,1 \mathrm{~g} / \mathrm{dm}^{3} \text {. }
\end{aligned}
$$

Le test de comparaison des moyennes conduit à un ( $t$ ) significatif de $-2,34$. Les arbres traités ont à très peu près la même densité que ceux de Mimizan au même âge ; les témoins, par contre, ont un bois sensiblement plus dense, en rapport vraisemblablement avec la moindre fertilité de la Station.

\section{4. - TEST D 441 (1965)}

Le dispositif D 441 fait partie des nombreuses plantations comparatives de descendances installées par la Station de Recherches forestières de Bordeaux en vue de l'amélioration génétique de cette essence.

Des études de densité du bois y ont été entreprises 3 ans après la mise en place de l'expérience, en sacrifiant un certain nombre de plants excédentaires destinés à d'éventuels regarnis. Les échantillons consistaient en fragments de tiges d'environ $3 \mathrm{~cm}$ de longueur prélevés à la partie inférieure des jeunes Pins, empattement déduit. Les densités calculées sont des infra-densités basées sur des déterminations de volume saturé faites au voluménomètre à mercure.

Il a déjà été rendu compte par ailleurs (ILLY, 1967) des résultats très importants obtenus en ce qui concerne l'héritabilité, au sens strict, de la densité du bois qui a pu être estimée à 0,56 par analyse des corrélations intra-classe dans les descendances, et à 0,54 par étude des régressions de la densité des arbres-mères à celle des descendants.

En fait, parmi les plants utilisés pour ces déterminations d'infra-densité, certains avaient bénéficié d'une assez bonne fertilisation de départ sous forme de $100 \mathrm{~g}$ d'hyperphosphate potassique 0-20-20 répandus au fond de chaque trou lors du repiquage des semis, alors que les autres, en nombre à peu près égal pour chaque famille, étaient destinés à servir de témoins et ne recevaient aucun apport d'engrais.

La comparaison des résultats obtenus séparément sur les plants fertilisés et nonfertilisés se présente comme suit : 
TABLEAU 4

\begin{tabular}{ll|c|c|c}
\hline \hline & & Témoins & Plants traités & Test « $t$ » \\
\cline { 2 - 4 } Nombre d'échantillons & $\ldots \ldots \ldots \ldots \ldots$ & 92 & 85 & \\
\hline Infra-densité $\left(\right.$ en $\left.\mathrm{g} / \mathrm{dm}^{3}\right)$ & $\ldots \ldots \ldots \ldots \ldots$ & 300,7 & 296,9 & $-1,3 \mathrm{NS}$ \\
\hline \hline
\end{tabular}

L'évolution de la densité se fait toujours dans le même sens, mais la diminution constatée à la suite de la fertilisation est faible (inférieure à $4 \mathrm{~kg}$ de matière sèche par $\mathrm{m}^{3}$ ), et non significative. Cette différence avec les expériences précédentes pourrait certes s'expliquer par une fertilité plus grande du milieu naturel; mais il semble bien que le plus jeune âge des plants lors des déterminations de densité soit, lui aussi, pour partie responsable de cet état de choses, et qu'on ait là un premier indice d'une variation dans le temps des effets de la fertilisation sur la structure du bois élaboré.

\section{5. - EXPÉRIENCE DE MIMIZAN. PRÉLÈVEMENTS DE 1966}

\section{1. - MATÉRIEL ET MÉTHODES}

Cette nouvelle étude, faite sur du matériel en provenance de la place d'expérience de Mimizan, a été entreprise à l'occasion de la première éclaircie qui y a été réalisée en 1966.

Malheureusement, comme c'est le plus souvent le cas dans une telle opération, on a cherché à dégager les meilleures tiges, en sorte que les sujets exploités sont loin de représenter la moyenne du peuplement.

On a dû prévoir, de ce fait, pour cette étude deux séries d'échantillons :

- les uns ont été prélevés sur les cinq plus grosses tiges enlevées en éclaircie dans chaque parcelle unitaire ; on pouvait penser qu'ils auraient, par suite, quelque chance de correspondre à des catégories de diamètre assez voisines de la moyenne pour chacune d'elles; malheureusement, les vérifications faites, imprécises faute de disposer d'un inventaire complet établi à partir de mesures faites à la même hauteur que les prélèvements, ont montré que les tiges en cause avaient encore des dimensions transversales inférieures à celles de l'arbre moyen dans les divers placeaux ;

- les autres ont été tirés d'un échantillonnage systématique qui a porté sur le premier plant exploité dans chaque ligne de numéro pair de chaque parcelle unitaire ; ils représentent valablement l'éclaircie, mais leurs accroissements sont obligatoirement inférieurs à ceux du reste du peuplement ; les premiers seront appelés ici échantillons «plus », les seconds, échantillons « systématiques $\gg$.

Deux caractéristiques physiques : la densité totale et la densité des trois derniers cernes, ainsi qu'une caractéristique auxométrique : la longueur radiale moyenne ont fait l'objet d'une première série d'analyses, puis ont été étudiées deux caractéristiques papetières : le rendement en fibres et la longueur moyenne des fibres.

Les échantillons reçus consistaient en morceaux de tiges de $3 \mathrm{~cm}$ de longueur environ prélevés a une dizaine de centimètres au-dessus du sol, de façon à éviter l'influence perturbatrice de l'empattement. Dans chacun d'eux ont été découpés deux secteurs cylindriques excluant à la fois la zone à larges cernes du côté est, responsable de l'excentricité du Pin maritime dans les Landes, ainsi que celle à cernes étroits qui lui est opposée.

C'est sur ces secteurs qu'ont été mesurés les rayons moyens et calculées les infra-densités à partir de volumes à l'état frais résultant de pesées successives dans l'air et dans l'eau; les trois derniers cernes ont ensuite été séparés et leur densité moyenne a été déterminée sur la base des volumes obtenus au voluménomètre à mercure.

Enfin, quelques fragments de bois d'été de l'année 1964, représentant environ $0,8 \mathrm{~g}$ de matière 
sèche, ont été défibrés dans des microlessiveurs suivant un procédé déjà décrit par ailleurs (PoLgE. 1967), ce qui a permis de connaitre le rendement en fibres et la longueur moyenne des fibres à un instant donné de la vie des arbres, identique pour tous les sujets étudiés.

\section{2. - RÉSULTATS}

\subsection{Densité globale, densité des trois derniers cernes et rayon moyen}

Ces trois caractéristiques ont fait l'objet de toute une série d'études statistiques qui ont été menées à bien par R. Tomassone et F. Labour de la Station de Biométrie du CNRF.

Dans un premier temps, elles ont été analysées séparément sur les échantillons plus et les échantillons systématiques, soit un total de 6 variables avec comme source de variation les 7 traitements du dispositif de Mimizan : c'est-à-dire les témoins $(\mathrm{T})$ et les traitements à Azote seul $(\mathrm{N})$, Azote et Potasse (NK), Phosphore seul (P), Phosphore et Azote (NP), Phosphore et Potasse (PK) et enfin fertilisation complète (NPK).

Par la suite, on a étudié, toujours pour les 6 variables ci-dessus indiquées, le contraste entre les témoins et, d'une part, l'ensemble des plants $\mathrm{N}$ et NK, et d'autre part, l'ensemble des plants P, NP, PK et NPK.

Enfin, dans une troisième étape, de nouvelles analyses de variance ont été faites en regroupant les échantillons plus et les échantillons systématiques, et en considérant la taille des plants comme une troisième source de variation, au même titre que le bloc ou le traitement.

Pour toutes ces investigations, le nombre d'individus étudiés n'a pas pu être identique pour les divers placeaux car, en particulier, il est arrivé qu'aucun plant ne soit enlevé en éclaircie dans l'une ou l'autre des lignes paires sur lesquelles portait l'échantillonnage systématique. De ce fait, on s'est trouvé, pour les analyses de variance, dans le cas de dispositif en blocs non orthogonaux (Tomassone, 1964).

\subsection{Comparaison traitement par traitement}

5.2111. Densité trois cernes échantillons ( plus))

Les résultats sont consignés dans le tableau 5 :

\section{TABLEAU 5}

Analyse de la variance

\begin{tabular}{|c|c|c|c|c|c|c|}
\hline \multirow{2}{*}{ Source de variation } & \multirow{2}{*}{$\begin{array}{l}\text { Somme des } \\
\text { carrés des } \\
\text { écarts }\end{array}$} & \multirow{2}{*}{$\begin{array}{l}\text { Degrés de } \\
\text { liberté }\end{array}$} & \multirow{2}{*}{$\begin{array}{l}\text { Carré } \\
\text { moyen }\end{array}$} & \multicolumn{3}{|c|}{$\mathrm{F}$} \\
\hline & & & & $\begin{array}{l}\text { Valeur } \\
\text { calculée }\end{array}$ & \begin{tabular}{|cc} 
& Valeur \\
Seuil & 0,05
\end{tabular} & $\begin{array}{l}\text { limite } \\
\text { Seuil } \quad 0,01\end{array}$ \\
\hline Blocs $\ldots \ldots \ldots$. & 1177,94 & 4 & 294,5 & $1,03 \mathrm{NS}$ & 2,87 & 4,43 \\
\hline Traitements ... & 2722,84 & 6 & 453,8 & $1,58 \mathrm{NS}$ & 2,60 & 3,87 \\
\hline Erreur $\ldots \ldots$ & 5728,91 & 20 & 286,4 & & & \\
\hline Totale $\ldots \ldots \ldots \ldots \ldots$ & 9629,69 & 30 & & & & \\
\hline
\end{tabular}


Comparaison des moyennes

\begin{tabular}{|c|c|c|c|c|c|c|c|}
\hline Traitement & $\mathrm{T}$ & NK & PK & $\mathrm{N}$ & $\mathrm{P}$ & NP & NPK \\
\hline $\begin{array}{r}\text { Moyenne ajustée (en } \\
\left.\mathrm{g} / \mathrm{dm}^{3}\right) \quad \ldots \ldots \ldots \ldots\end{array}$ & 403,7 & 388,5 & 381,5 & 376,8 & 375,7 & 375,5 & 369,5 \\
\hline $\begin{array}{l}\text { Test de Duncan } \\
\text { (seuil } 0,05 \text { ) }\end{array}$ & & & & 1 & & & \\
\hline
\end{tabular}

Globalement, la variance entre traitements n'est pas significativement supérieure à celle de l'erreur ; mais le test de Duncan montre qu'il existe une différence significative au seuil de $5 \%$ entre les placeaux témoins et ceux ayant bénéficié de phosphore, à l'exception des placeaux PK.

\subsection{Densité trois cernes échantillons ( systématiques )}

\section{TABLEAU 6}

Analyse de la variance

\begin{tabular}{|c|c|c|c|c|c|c|}
\hline \multirow{3}{*}{ Source de variation } & \multirow{3}{*}{$\begin{array}{l}\text { Somme des } \\
\text { carrés des } \\
\text { écarts }\end{array}$} & \multirow{3}{*}{$\underset{\text { liberté }}{\text { Degrés de }}$} & \multirow{3}{*}{$\begin{array}{l}\text { Carré } \\
\text { moyen }\end{array}$} & \multicolumn{3}{|c|}{$\mathrm{F}$} \\
\hline & & & & Valeur & Valeur & limite \\
\hline & & & & calculée & Seuil 0,05 & Seuil $\quad 0,01$ \\
\hline Blocs $\ldots \ldots \ldots \ldots \ldots$. & 967,44 & 4 & 241,9 & $1,95 \mathrm{NS}$ & 2,90 & 4,50 \\
\hline Traitements $\ldots \ldots \ldots$ & 2113,03 & 6 & 352,2 & $2,83^{*}$ & 2,63 & 3,94 \\
\hline Erreur $\ldots \ldots \ldots \ldots$ & 2366,91 & 19 & 124,6 & & & \\
\hline Totale $\ldots . \ldots \ldots \ldots$. & 5447,38 & 29 & & & & \\
\hline
\end{tabular}

Comparaison des moyennes

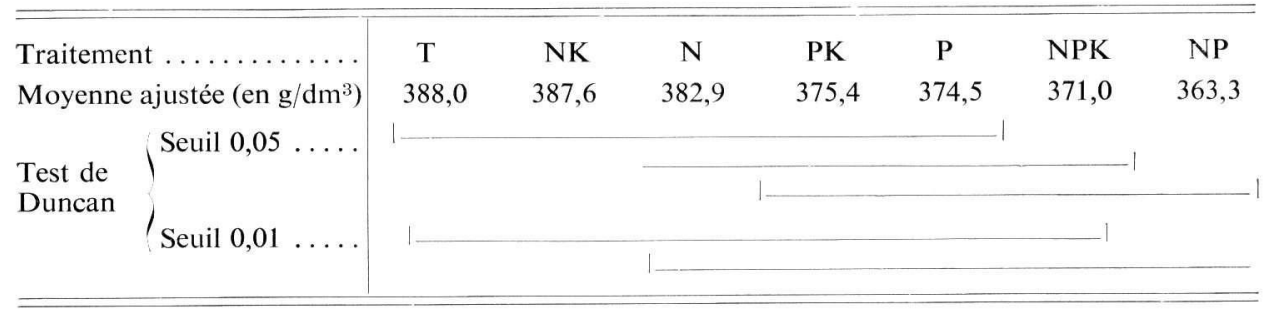

Il existe maintenant des différences globales entre traitements ; en étudiant ceux-ci séparément, on voit qu'elles se manifestent surtout entre témoins d'une part, NP et NPK de l'autre ; les quatre séries de placeaux ayant bénéficié d'engrais phosphaté arrivent derniers dans l'ordre des densités décroissantes, mais au seuil $5 \%$, la plupart d'entre eux ne diffèrent pas des placeaux $\mathrm{N}$.

Les écarts avec les échantillons ( plus ) sont dans l'ensemble faibles, sauf pour les témoins où l'importance de la différence constatée laisse à nouveau penser à une influence probable de facteurs génétiques. 
5.2113. Densité totale échantillons " plus »

\section{TABLEAU 7}

Analyse de la variance

\begin{tabular}{|c|c|c|c|c|c|c|c|c|}
\hline \multirow{3}{*}{ Source de variation } & \multirow{3}{*}{$\begin{array}{l}\text { Somme des } \\
\text { carrés des } \\
\quad \text { écarts }\end{array}$} & \multirow{3}{*}{$\begin{array}{c}\text { Degrés de } \\
\text { liberté }\end{array}$} & \multirow{3}{*}{$\begin{array}{l}\text { Carré } \\
\text { moyen }\end{array}$} & \multicolumn{5}{|c|}{$\mathrm{F}$} \\
\hline & & & & \multirow{2}{*}{$\begin{array}{l}\text { Valeur } \\
\text { calculée }\end{array}$} & \multicolumn{4}{|c|}{ Valeur limite } \\
\hline & & & & & Seuil & 0,05 & Seuil & 0,01 \\
\hline Blocs ... & 642,25 & 4 & 160,6 & $0,57 \mathrm{NS}$ & 2 , & & 4, & \\
\hline Traitements ....... & 1456,64 & 6 & 242,8 & $0,86 \mathrm{NS}$ & 2 , & & 3, & \\
\hline Erreur & 5622,05 & 20 & 281,1 & & & & & \\
\hline Totale .......... & 7720,94 & 30 & & & & & & \\
\hline
\end{tabular}

Comparaison des moyennes

\begin{tabular}{|c|c|c|c|c|c|c|c|}
\hline Traitement $\ldots \ldots \ldots \ldots \ldots$ & $\mathrm{T}$ & NK & PK & $\mathrm{N}$ & NP & $\mathrm{P}$ & NPK \\
\hline Moyenne ajustée (en $\mathrm{g} / \mathrm{dm}^{3}$ ) & 388,0 & 376,0 & 373,8 & 369,1 & 366,4 & 365,0 & 364,8 \\
\hline $\begin{array}{l}\text { Test de Duncan } \ldots \ldots \ldots \ldots \\
\quad(\text { seuil } 0,05)\end{array}$ & & & & & & & \\
\hline
\end{tabular}

Il n'existe aucune différence significative entre traitements, ce qui confirme, par comparaison avec les résultats du paragraphe 5.2111., que les écarts provoqués par la fertilisation augmentent avec l'âge des plants (jusqu'à une limite qu'il conviendra de préciser ultérieurement).

\subsection{Densité totale échantillons (" systématiques )}

\section{TABleau 8}

Analyse de la variance

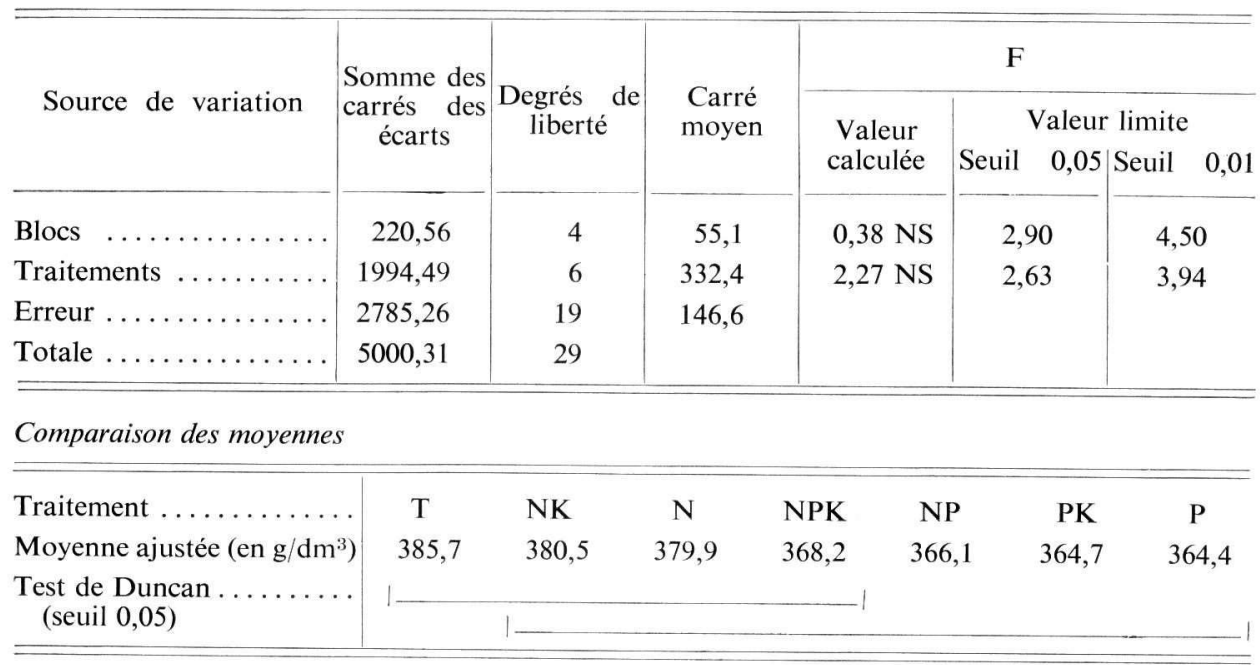


Pour tous les traitements (sauf NP), les densités totales sont inférieures à celles des trois derniers cernes, à cause de l'influence des accroissements voisins de la moelle dans lesquels la proportion de bois dense est peu élevée.

Les seules différences significatives qui existent le sont au seuil de $5 \%$, et cette plus grande homogénéité que l'on observe en passant, sur le même matériel, de la densité des seuls cernes 1963, 1964 et 1965 à la densité globale confirme la remarque faite pour les échantillons « plus » au paragraphe précédent.

\subsection{Rayon moyen échantillons ( plus )}

\section{TABLEAU 9}

Analyse de la variance

\begin{tabular}{|c|c|c|c|c|c|c|}
\hline \multirow{3}{*}{ Source de variation } & \multirow{3}{*}{$\begin{array}{l}\text { Somme des } \\
\text { carrés des } \\
\text { écarts }\end{array}$} & \multirow{3}{*}{$\begin{array}{l}\text { Degrés de } \\
\text { liberté }\end{array}$} & \multirow{3}{*}{$\begin{array}{l}\text { Carré } \\
\text { moyen }\end{array}$} & \multicolumn{3}{|c|}{$\mathrm{F}$} \\
\hline & & & & \multirow{2}{*}{$\begin{array}{l}\text { Valeur } \\
\text { calculée }\end{array}$} & \multicolumn{2}{|c|}{ Valeur limite } \\
\hline & & & & & Seuil 0,05 & Seuil \\
\hline Blocs & 1031240 & 4 & 257810 & $1,32 \mathrm{NS}$ & 2,87 & 4,43 \\
\hline Traitements ......... & 22541511 & 6 & 3756918 & $19,31 * *$ & 2,60 & 3,87 \\
\hline Erreur $\ldots \ldots \ldots \ldots \ldots$ & 3891513 & 20 & 194576 & & & \\
\hline Totale $\ldots \ldots \ldots \ldots$ & 27464264 & 30 & & & & \\
\hline
\end{tabular}

Comparaison des moyennes

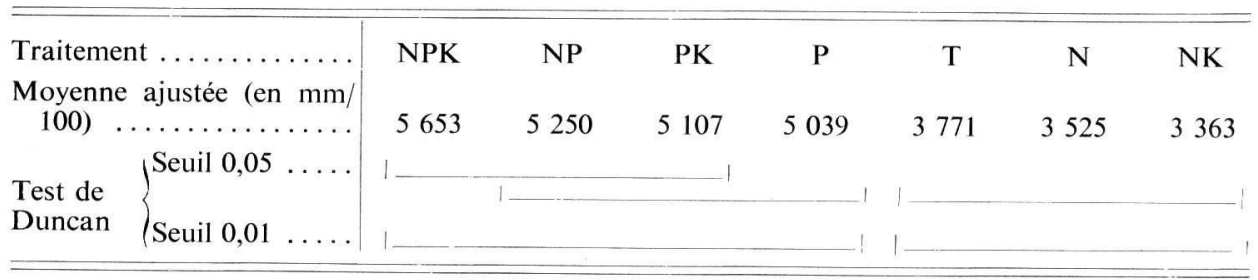

On retrouve ici des résultats maintenant bien connus en ce qui concerne les effets de la fertilisation sur la croissance des jeunes Pins; ils confirment, avec peut-être plus de netteté, ceux déjà publiés par Guinaudeau et $a l$. (op. cit.) pour la pousse en hauteur. On a ici en effet, au seuil de $1 \%$, une cassure brutale en deux groupes n'ayant pas de différences internes significatives : les arbres traités au Phosphore (seul ou en combinaison avec d'autres éléments fertilisants) et l'ensemble des autres.

Une autre remarque s'impose : en calculant les suppléments d'accroissement obtenus grâce aux engrais, on obtient les chiffres suivants (qui ne sont donnés ici qu'en rappellant que les échantillons (plus ) eux-mêmes avaient une croissance inférieure à la moyenne des placeaux) :

$\begin{array}{cccccc}\mathrm{NK} & \mathrm{N} & \mathrm{P} & \mathrm{PK} & \mathrm{NP} & \mathrm{NPK} \\ -\frac{11 \%}{\text { non significatifs }} & -6 \% & +34 \% & +35 \% & +39 \% & +50 \%\end{array}$


Si l'on se reporte à l'ouvrage cité plus haut, les gains obtenus pour la hauteur totale des plants, au terme de la $6^{\mathrm{e}}$ année, étaient les suivants (page 35 ) :

\begin{tabular}{|c|c|c|c|c|c|}
\hline $\mathrm{N}$ & NK & P & NP & PK & NPK \\
\hline$-14 \%$ & $-7 \%$ & $+54 \%$ & $+67 \%$ & $+69 \%$ & $+76 \%$ \\
\hline
\end{tabular}

Le parallélisme des résultats est frappant : l'ordre des divers traitements est identique, à l'exception de deux interversions minimes entre $\mathrm{N}$ et $\mathrm{NK}$ d'une part, NP et PK de l'autre. Seule surprend quelque peu la différence plus nette (mais non significative) constatée entre NPK et les autres traitements au phosphore. Par contre, on doit souligner le fait que, dans les deux cas, l'apport d'azote, seul ou en combinaison avec la potasse, a entraîné une baisse de croissance, non significative il est vrai, aux seuils habituellement choisis ; mais le calcul montre qu'il y a, par exemple, 80 chances sur 100 pour que la réduction de l'accroissement sur le rayon, constatée dans les placeaux NK, ne soit pas due au hasard.

\subsection{Comparaison entre témoins et groupes de traitements}

Les analyses précédentes ayant montré que les différences significatives étaient très rares entre les divers traitements comportant un apport de phosphore (seul ou en combinaison), et nulles entre les placeaux $\mathrm{N}$ et $\mathrm{NK}$, de nouveaux tests de comparaison de moyennes ont été calculés entre les trois groupes : témoins, ensemble $\mathrm{P}, \mathrm{NP}$, PK et NPK, et ensemble $\mathrm{N}$ et NK pris deux à deux. Les résultats sont les suivants :

Tableau 10

\begin{tabular}{|c|c|c|c|c|c|c|}
\hline & \multicolumn{2}{|c|}{ Contraste Témoin - P } & \multicolumn{2}{|c|}{ Contraste Témoin - N } & \multicolumn{2}{|c|}{ Contraste $P-N$} \\
\hline & $\begin{array}{c}\text { Différence des } \\
\text { moyennes } \\
\text { ajustées }\end{array}$ & $\begin{array}{l}\text { Test } \\
\langle t\rangle\end{array}$ & $\begin{array}{l}\text { Différence des } \\
\text { moyennes } \\
\text { ajustées }\end{array}$ & $\begin{array}{l}\text { Test } \\
\langle t »\end{array}$ & $\begin{array}{l}\text { Différence des } \\
\text { moyennes } \\
\text { ajustées }\end{array}$ & $\begin{array}{l}\text { Test } \\
« t »\end{array}$ \\
\hline $\begin{array}{c}\text { Densité } 3 \text { cernes } \\
\text { échantillons } \\
\text { «plus }(20 \text { D.L. })\end{array}$ & $28,2 \mathrm{~g} / \mathrm{dm}^{3}$ & $2,61^{*}$ & $21,1 \mathrm{~g} / \mathrm{dm}^{3}$ & $1,77 \mathrm{NS}$ & $7,1 \mathrm{~g} / \mathrm{dm}^{3}$ & $0,97 \mathrm{NS}$ \\
\hline $\begin{array}{c}\text { Densité } 3 \text { cernes } \\
\text { échantillons } \\
\text { "systématiques» } \\
\text { (19 D.L.) ..... }\end{array}$ & $17,0 \mathrm{~g} / \mathrm{dm}^{3}$ & $2,73^{*}$ & $2,8 \mathrm{~g} / \mathrm{dm}^{3}$ & $0,40 \mathrm{NS}$ & $14,2 \mathrm{~g} / \mathrm{dm}^{3}$ & $2,89 * *$ \\
\hline $\begin{array}{l}\text { Densité totale } \\
\text { échantillons } \\
\text { «plus }(20 \text { D.L. })\end{array}$ & $20,4 \mathrm{~g} / \mathrm{dm}^{3}$ & $1,91 \mathrm{NS}$ & $15,4 \mathrm{~g} / \mathrm{dm}^{3}$ & 1,31 NS & $5,1 \mathrm{~g} / \mathrm{dm}^{3}$ & $0,69 \mathrm{NS}$ \\
\hline $\begin{array}{l}\text { Densité totale } \\
\text { échantillons } \\
\text { ((systématiques) } \\
\text { (19 D.L.)..... }\end{array}$ & $19,8 \mathrm{~g} / \mathrm{dm}^{3}$ & $2,91 * *$ & $5,4 \mathrm{~g} / \mathrm{dm}^{3}$ & $0,72 \mathrm{NS}$ & $14,4 \mathrm{~g} / \mathrm{dm}^{3}$ & $2,71^{*}$ \\
\hline $\begin{array}{l}\text { Rayon moyen } \\
\text { échantillons } \\
\text { «plus » }(20 \text { D.L. })\end{array}$ & $14,91 \mathrm{~mm}$ & $5,30^{* *}$ & $3,27 \mathrm{~mm}$ & $1,06 \mathrm{NS}$ & $18,18 \mathrm{~mm}$ & $9,47 * *$ \\
\hline $\begin{array}{l}\text { Rayon moyen } \\
\text { échantillons } \\
\text { ((systématiques)) } \\
\text { (19 D.L.)..... }\end{array}$ & $11,70 \mathrm{~mm}$ & $3,94 * *$ & $0,71 \mathrm{~mm}$ & $0,21 \mathrm{NS}$ & $10,99 \mathrm{~mm}$ & $4,70 * *$ \\
\hline
\end{tabular}


Les tendances entrevues précédemment se trouvent confirmées : on n'a aucune différence significative entre les témoins et les arbres ayant bénéficié d'un apport d'azote ou de potasse, mais sans phosphore.

Pour le reste, on observe des différences significatives au seuil de 1 ou de $5 \%$, aussi bien entre phosphore et témoins qu'entre phosphore et azote pour tout ce qui concerne la croissance radiale d'une part, et les densités totales, ou mesurées seulement sur les trois derniers cernes, des échantillons systématiques d'autre part ; enfin, l'effet des engrais apparaît beaucoup plus faible lorsqu'on a affaire aux échantillons plus puisqu'on ne relève plus qu'une seule différence significative limitée au seuil de $5 \%$ : celle qui intéresse le contraste de densité entre phosphore et témoins sur les trois derniers cernes.

\subsection{Comparaison portant sur l'ensemble des échantillons plus et des échantillons systématiques}

Dans une dernière étude, la Station de Biométrie du CNRF a procédé à une analyse factorielle à 3 facteurs contrôlés : le facteur bloc (à 5 niveaux), le facteur traitement (à 7 niveaux) et enfin le facteur dimension à 2 niveaux (échantillons plus et échantillons systématiques).

\subsection{Comparaison des densités des trois derniers cernes}

L'analyse de variance se présente comme suit :

TABLEAU 11

\begin{tabular}{|c|c|c|c|c|c|c|}
\hline \multirow{3}{*}{ Source de variation } & \multirow{3}{*}{$\begin{array}{l}\text { Somme des } \\
\text { carrés des } \\
\text { écarts }\end{array}$} & \multirow{3}{*}{$\begin{array}{c}\text { Degrés de } \\
\text { liberté }\end{array}$} & \multirow{3}{*}{$\begin{array}{l}\text { Carré } \\
\text { moyen }\end{array}$} & \multicolumn{3}{|c|}{$\mathrm{F}$} \\
\hline & & & & Valeur & Valeur & limite \\
\hline & & & & calculée & Seuil $\quad 0,05$ & Seuil $\quad 0,01$ \\
\hline Bloc (A) $\ldots \ldots \ldots$ & 2178,50 & 4 & 544,6 & $4,09^{*}$ & 2,78 & 4,22 \\
\hline Traitement (B) & 5499,37 & 6 & 916,6 & $6,89 * *$ & 2,51 & 3,67 \\
\hline Dimension (C) & 488,88 & 1 & 488,9 & $3,67 \mathrm{NS}$ & 4,26 & 7,82 \\
\hline Interaction $\mathrm{AB} \ldots$ & 4914,50 & 24 & 204,8 & 1,54 NS & 1,98 & 2,66 \\
\hline Interaction $\mathrm{BC} \ldots$ & 940,37 & 6 & 156,7 & $6,53 * *$ & & \\
\hline Interaction CA ... & 632,50 & 4 & 158,1 & 1,19 NS & & \\
\hline Erreur...$\ldots \ldots \ldots$ & 3193,75 & 24 & 133,1 & & & \\
\hline Total $\ldots \ldots \ldots \ldots$ & 17847,87 & 69 & & & & \\
\hline
\end{tabular}

Il y a un léger effet bloc, et surtout une différence très significative entre traitements ; l'analyse de variance ne permet pas de mettre en évidence une influence significative du facteur dimension, et cependant, l'interaction des effets traitement et dimension est très significative, ce qui confirme que l'influence du traitement n'est pas la même selon que l'on a affaire à des échantillons plus ou à des échantillons systématiques. 
Si néanmoins on regroupe ensemble ces deux catégories, la comparaison des moyennes se présente comme suit :

TABLEAU 12

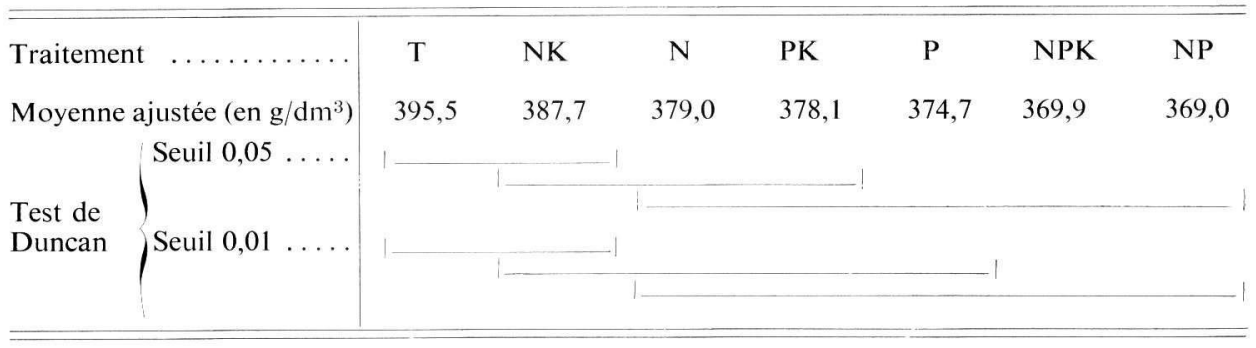

5.2132. Comparaison des densités totales

TABleau 13

\begin{tabular}{|c|c|c|c|c|c|c|}
\hline \multirow{3}{*}{ Source de variation } & \multirow{3}{*}{$\begin{array}{l}\text { Somme des } \\
\text { carrés des } \\
\text { écarts }\end{array}$} & \multirow{3}{*}{$\begin{array}{c}\text { Degrés de } \\
\text { liberté }\end{array}$} & \multirow{3}{*}{$\begin{array}{l}\text { Carré } \\
\text { moyen }\end{array}$} & \multicolumn{3}{|c|}{$\mathrm{F}$} \\
\hline & & & & Valeur & Valeur & limite \\
\hline & & & & calculée & Seuil $\quad 0,05$ & Seuil $\quad 0,01$ \\
\hline Bloc (A) ......... & 1362,50 & 4 & 340,6 & $2,18 \mathrm{NS}$ & 2,78 & 4,22 \\
\hline Traitement (B) $\ldots$ & 4026,12 & 6 & 671,0 & $4,29 * *$ & 2,51 & 3,67 \\
\hline Dimension (C) .. & 2,50 & 1 & 2,5 & $0,02 \mathrm{NS}$ & 4,26 & 7,82 \\
\hline Interaction $\mathrm{AB} \ldots$ & 4357,50 & 24 & 181,6 & $1,16 \mathrm{NS}$ & 1,98 & 2,66 \\
\hline Interaction $\mathrm{BC} \ldots$ & 670,75 & 6 & 111,8 & $4,66^{* *}$ & & \\
\hline Interaction $\mathrm{CA} \ldots \ldots$ & 303,00 & 4 & 75,7 & $0,48 \mathrm{NS}$ & & \\
\hline Erreur ........... & 3756,25 & 24 & 156,5 & & & \\
\hline Total ........... & 14478,62 & 69 & & & & \\
\hline
\end{tabular}

L'effet bloc n'est maintenant plus significatif, non plus que l'effet dimension ; l'influence du traitement demeure très significative, de même que l'interaction entre traitement et dimension.

En regroupant cependant échantillons plus et échantillons systématiques, on a :

TABleau 14

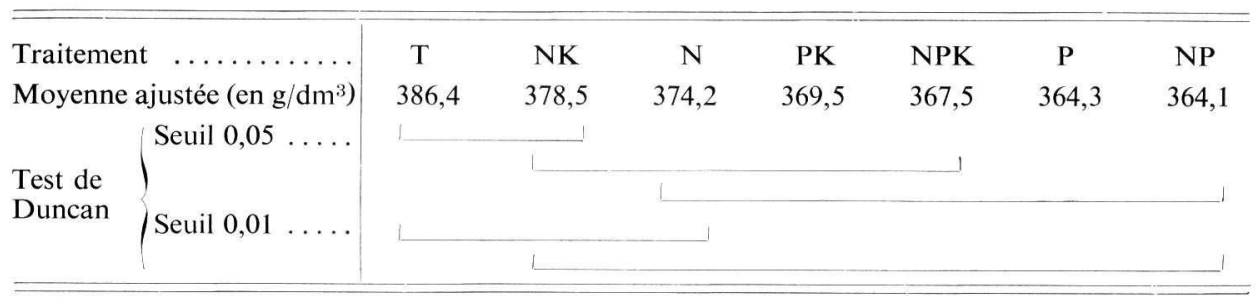


On retrouve maintenant des résultats bien connus, et en particulier, les seules différences significatives qui subsistent au seuil 0,01 sont celles que l'on observe entre témoins d'une part, et l'ensemble des arbres ayant bénéficié de phosphore d'autre part.

\subsection{Comparaison entre rayon moyen}

\section{Tableau 15}

\begin{tabular}{|c|c|c|c|c|c|c|}
\hline \multirow{3}{*}{ Source de variation } & \multirow{3}{*}{$\begin{array}{l}\text { Somme des } \\
\text { carrés des } \\
\text { écarts }\end{array}$} & \multirow{3}{*}{$\begin{array}{l}\text { Degrés de } \\
\text { liberté }\end{array}$} & \multirow{3}{*}{$\begin{array}{l}\text { Carré } \\
\text { moyen }\end{array}$} & \multicolumn{3}{|c|}{$\mathrm{F}$} \\
\hline & & & & \multirow{2}{*}{$\begin{array}{l}\text { Valeur } \\
\text { calculée }\end{array}$} & \multicolumn{2}{|c|}{ Valeur limite } \\
\hline & & & & & Seuil 0,05 & Seuil \\
\hline Bloc (A) & 3042608 & 4 & 760652 & $3,66^{*}$ & 2,78 & 4,22 \\
\hline Traitement (B) & 37941312 & 6 & 6323552 & $30,43^{* * *}$ & 2,51 & 3,67 \\
\hline Dimension (C) & 23454128 & 1 & 23454128 & $112,88 * *$ & 4,26 & 7,82 \\
\hline Interaction $\mathrm{AB} \ldots$ & 4279088 & 24 & 178295 & $0,86 \mathrm{NS}$ & 1,98 & 2,66 \\
\hline Interaction $\mathrm{BC} \ldots$ & 2142512 & 6 & 357085 & $14878,56^{* * *}$ & & \\
\hline Interaction CA ... & 1355888 & 4 & 338972 & $1,63 \mathrm{NS}$ & & \\
\hline Erreur $\ldots \ldots \ldots \ldots \ldots$ & 4986800 & 24 & 207783 & & & \\
\hline Total $\ldots \ldots \ldots \ldots$. & 77202336 & 69 & & & & \\
\hline
\end{tabular}

On a, comme on pouvait s'y attendre, un effet dimension très significatif et il s'y ajoute un léger effet bloc; la comparaison des moyennes en regroupant échantillons plus et échantillons systématiques n'a dès lors plus grande signification et n'est donnée ci-dessous qu'à titre de simple information :

\section{TABLEAU 16}

\begin{tabular}{|c|c|c|c|c|c|c|c|}
\hline Traitement $\ldots \ldots \ldots \ldots \ldots$ & NPK & NP & $\mathbf{P}$ & PK & $\mathrm{N}$ & $\mathrm{T}$ & NK \\
\hline $\begin{array}{c}\text { Moyenne ajustée (en } \mathrm{mm} / \\
100) \ldots \ldots \ldots \ldots \ldots \ldots\end{array}$ & 5014,2 & $443 !, 4$ & 4391,5 & 4382,5 & 3274,8 & 3224,2 & 2917,9 \\
\hline Test de Duncan (seuil 0,01). & $1 \ldots$ & 1 & & $\ldots$ & & & \\
\hline
\end{tabular}

Notons cependant que c'est ici la première fois, aussi bien dans cette étude que dans les précédentes consacrées à l'expérience de Mimizan, qu'une différence très significative apparaît entre les arbres ayant bénéficié d'une fertilisation complète et les autres arbres fertilisés.

\subsection{Caractéristiques papetières}

Elles ont été déterminées sur un échantillonnage réduit comportant 18 témoins, 18 arbres ayant bénéficié d'un apport de phosphore, et 18 autres ayant bénéficié d'un apport d'azote et de potasse, tirés au hasard dans les divers blocs du dispositif ; les études statistiques correspondantes ont été menées à bien par C. MILLIER de la Station de Biométrie du CNRF. 


\subsection{Rendement en fibres}

L'analyse de variance conduit aux résultats suivants :

TABLEAu 17

\begin{tabular}{c|c|c|c|c}
\hline \hline \multicolumn{1}{c|}{ Source de variation } & $\begin{array}{c}\text { Somme des } \\
\text { carrés des } \\
\text { écarts }\end{array}$ & $\begin{array}{c}\text { Degrés de } \\
\text { liberté }\end{array}$ & $\begin{array}{c}\text { Carré } \\
\text { moyen }\end{array}$ & F \\
\hline Traitements $\ldots \ldots \ldots \ldots \ldots \ldots \ldots$ & 20,26 & 2 & 10,13 & 2,06 NS \\
Erreur $\ldots \ldots \ldots \ldots \ldots \ldots \ldots \ldots$ & 250,79 & 51 & 4,92 & \\
Total $\ldots \ldots \ldots \ldots \ldots \ldots \ldots \ldots$ & 271,04 & 53 & $\ldots \ldots \ldots$ & \\
\hline \hline
\end{tabular}

Il n'y a pas de différence significative globale entre traitement, mais si l'on procède à un test de comparaison de moyennes, on obtient les résultats suivants :

TABleau 18

\begin{tabular}{l|c|c|c}
\hline \hline Traitement $\ldots \ldots \ldots \ldots \ldots \ldots \ldots \ldots \ldots \ldots$ & P & NK & T \\
Moyenne $($ en $\%$ ) $\quad \ldots \ldots \ldots \ldots \ldots \ldots \ldots \ldots$ & 46,16 & 45,38 \\
Test de Duncan (seuil $5 \% \ldots \ldots \ldots \ldots \ldots$ & & & 44,67 \\
& & \\
\hline
\end{tabular}

On observe donc, pour les arbres à phosphore, une amélioration significative au seuil de $5 \%$ dans le rendement en fibres (reporté au poids sec de bois), et ce gain vient contrebalancer en partie la perte constatée au point de vue de la densité.

\subsection{Longueur des fibres}

Pour chacun des 54 arbres-échantillons précédemment utilisés pour la détermination des rendements en fibres, ont été établies des courbes de fréquence de longueur de fibres basées sur la mensuration au curvimètre de 100 fibres par échantillon en employant un rapport d'agrandissement égal à 100 .

\subsection{Test de normalité et transformation}

A partir des diagrammes de répartition a été contrôlée la normalité de la distribution des longueurs de fibres pour un certain nombre d'échantillons en appliquant aux pourcentages de fréquence cumulés le test de la droite de Henry.

Cette vérification a permis de constater que la répartition des longueurs de fibres mesurées n'était pas normale ; on a des courbes dissymétriques avec un net déficit de fibres de longueur inférieure à la moyenne ; on peut penser que cet état de choses provient du fait que les Techniciens chargés des mesures ont tendance à considérer les fibres courtes comme incomplètes, et donc à ne pas les prendre en compte, alors qu'au contraire toutes les fibres longues sont systématiquement mesurées. 
Quoi qu'il en soit, une transformation logarithmique améliore considérablement la normalité, ainsi que l'on peut s'en rendre compte en calculant les coefficients $\mathrm{b}_{1}$ de Pearson, qui permettent de tester les écarts à la loi normale et qui sont nuls en cas de distribution parfaitement gaussienne : sur 54 échantillons, on avait, avant transformation, 15 coefficients $b_{1}$ différents de 0 au seuil de $1 \%$ et 10 au seuil de $5 \%$; après transformation logarithmique, ces chiffres sont ramenés à 2 et 4 échantillons respectivement.

Par ailleurs, l'homogénéité de la variance se trouve grandement améliorée, puisque celle-ci ne varie plus que du simple au double, alors qu'avant transformation, l'amplitude des variations était de 1 à plus de 4 .

Quelles que soient les raisons des écarts constatés par rapport à la normalité, la transformation logarithmique des longueurs de fibres s'impose donc, et permet une analyse plus précise de la variabilité.

\subsection{Analyse de variance}

Elle se trouve résumée dans le tableau suivant :

TABLEAu 19

\begin{tabular}{|c|c|c|c|c|}
\hline Origine de la variation & $\begin{array}{l}\text { Somme des } \\
\text { carrés }\end{array}$ & d.I. & Carré moyen & F \\
\hline Entre traitements $\ldots \ldots \ldots \ldots$ & 6,9336 & 2 & 3,3765 & $2,12 \mathrm{NS}$ \\
\hline $\begin{array}{l}\text { Entre arbres au sein d'un trai- } \\
\text { tement } \ldots \ldots \ldots \ldots \ldots \ldots\end{array}$ & 80,69 & 61 & 1,5821 & $69,96 * *$ \\
\hline Erreur $\ldots \ldots \ldots \ldots \ldots \ldots$ & 121,5524 & 5382 & 0,226 & \\
\hline Total $\ldots \ldots \ldots \ldots \ldots$ & & 5399 & & \\
\hline
\end{tabular}

On voit que, comme on pouvait s'y attendre, la variabilité est bien plus grande au niveau individuel entre arbres qu'entre traitements, et que, globalement, l'influence de la fertilisation sur la longueur moyenne des fibres n'est pas significative.

Le test de comparaison de moyennes permet d'aboutir aux résultats suivants :

TABleau 20

\begin{tabular}{lr|ccc}
\hline \hline Traitement $\ldots \ldots \ldots \ldots \ldots \ldots \ldots \ldots \ldots$ & P & NK & T \\
Moyenne $($ en $\mathrm{mm} / 100) \ldots \ldots \ldots \ldots \ldots \ldots \ldots$ & 193,79 & 191,08 & 178,16 \\
Test de Duncan $($ seuil $5 \% \ldots \ldots \ldots \ldots \ldots \ldots$ & & \\
\hline \hline
\end{tabular}

Là encore, on ne retrouve pas de différence significative au niveau traitement, mais on doit cependant noter une tendance assez nette à un allongement des fibres dans les placeaux ayant bénéficié de la fertilisation. 


\section{CONCLUSION}

Les diverses expériences dont il est rendu compte ici ont confirmé un certain nombre de résultats déjà enregistrés lors d'études analogues faites à l'étranger.

C'est le cas notamment de la baisse de densité du bois liée à l'accélération de la croissance que provoque une fertilisation réussie ; de nombreux auteurs l'ont observée dans un passé récent, parmi lesquels ERICKSON et LAMBERT (1958), Zobel et al. (1961), Williams et Hamilton (1961), Posey (1964)...

Mais le présent travail a permis en outre d'obtenir, sous les réserves formulées plus haut en ce qui concerne certains échantillonnages, quelques informations complémentaires nouvelles:

D'une part, les courbes de variations de la densité du bois ont montré que la fertilisation réduit davantage les densités maximales que les densités minimales annuelles, d'où résulte une diminution du contraste de densité à l'intérieur des cernes et, par suite, une moindre hétérogénéité du bois ; de plus, une réduction très significative du pourcentage de bois de densité supérieure à $500 \mathrm{~g} / \mathrm{dm}^{3}$ a été observée, et la baisse constatée au niveau $600 \mathrm{~g} / \mathrm{dm}^{3}$ est elle-même très près du seuil de signification.

D'autre part, plusieurs indices concordants semblent indiquer une augmentation avec le temps de l'effet négatif des engrais sur la densité du bois, ce qui est contraire à l'évolution que POSEY (op. cit.) a mise en évidence sur Pinus taeda ; ici une aggravation sensible de la perte en densité du bois constatée sur des arbres ayant bénéficié d'une fertilisation phosphatée par rapport aux témoins apparaît clairement dans l'expérience de Mimizan : la baisse de densité était en effet de 1,7\% en 1964, alors que le matériel d'étude était constitué par des carottes de sondage où les cernes les plus anciens, c'est-à-dire les plus voisins de la moelle, sont sur-représentés en raison de la forme cylindrique des prélèvements ; elle s'est élevée à 6,3\% en 1966 lorsqu'on a utilisé comme matériel d'étude des secteurs cylindriques complets (où la surface des divers accroissements annuels est proportionnelle à celle qu'ils occupent en réalité dans l'arbre au niveau considéré), et enfín, à 7,4\% lorsque l'on n'a pris en considération que les trois cernes les plus récemment produits.

Dans les autres domaines touchant à la qualité du bois, on doit noter une augmentation non significative du retrait longitudinal, et surtout un certain nombre de modifications touchant aux caractéristiques papetières :

- le rendement en fibres (rapporté au poids sec de bois) des arbres ayant bénéficié de phosphore est supérieur de 3,3\% à celui des témoins, et cette augmentation (rendement passant de $44,67 \%$ à $46,16 \%$ ) est significative ;

- en ce qui concerne la longueur des fibres (facteur très important qui conditionne la résistance à la déchirure des papiers), celle des arbres ayant reçu du phosphore est supérieure de $8,7 \%$ à celle des témoins, et le moins qu'on puisse dire est que l'on n'a pas retrouvé ici l'influence néfaste de la fertilisation sur la longueur des trachéides mise en évidence par plusieurs auteurs, et notamment par ZoBEL (op. cit.) et DADSWell (1963). 
$\mathrm{Au}$ total, des diverses modifications occasionnées par les apports d'engrais, la plus inquiétante est sans doute la baisse de la densité qui paraît aller en s'aggravant au fur et à mesure que les peuplements en cause vieillissent ; cette baisse n'est cependant pas jusqu'à maintenant trop considérable, et ne saurait, en aucun cas, contrebalancer les gains de croissance très importants qui ont pu être obtenus.

Il reste à savoir si l'évolution constatée se maintiendra à l'avenir, et surtout si des différences, jusqu'à maintenant non significatives, les unes favorables (réduction de l'hétérogénéité du bois, augmentation des longueurs de fibres), les autres défavorables (aggravation du retrait longitudinal) ne tendront pas à le devenir ultérieurement.

Reşu pour publication en septembre 1968

\section{REMERCIEMENTS}

Plusieurs spécialistes de la Station de Biométrie nommément désignés plus haut ont procédé aux études statistiques dont il est rendu compte ici.

Par ailleurs, J. Guinaudeau et G. Illy de la Station de Recherches de Bordeaux, ainsi que J. Bulle des Papeteries de Gascogne ont recueilli, et nous ont fait parvenir, une partie des échantillons utilisés dans ce travail.

Que les uns et les autres trouvent ici l'expression de nos bien vifs remerciements.

\section{SUMMARY \\ EFFECT OF FERTILIZATION UPON THE WOOD PROPERTIES of Pinus pinaster}

Several studies concerning Pinus Pinaster of the Landes Forest treated with mineral fertilizers have shown that bringing Phosphorus in particular results in an improvement of radial growth and a decrease of wood density which is generally significant.

This last mentioned effect seems to become more important as the number of years since the fertilization increases :

In the same experiment and for the same treatment, the loss of specific gravity is $1,7 \%$ at the end of the 7th year (table 2) with, as samples, increment cores in which the low-density rings near the pith are over-represented, $6,3 \%$ at the end of the 9th year for the total stem section (table 7 ) and $7,4 \%$ at the same age for the 3 last rings only (table 5).

The study of density variations within rings shows (table 3 ) that the maximum annual densities are more reduced than the minimum ones so that the heteregoneity of wood decreases; elsewhere, the percentage of dense wood within rings is significantly lower in the trees treated with phosphorus.

The longitudinal shrinkage is higher after fertilization but the difference is not significant (table 1). The pulp yield in the plots with phosphorus is significantly higher than in the control plots (table 17).

At last, contrarily to what is reported in the litterature, the fiberlength is increased in fertilized trees, but not significantly.

\section{ZUSAMMENFASSUNG}

Einfluss der düngung auf die Holzqualität der Seestrandkiefer vON Pinus pinaster

Verschiedene Untersuchungen an gedungten Seestrandkiefern in den « Landes » ergaben, dass insbesondre eine Phosphordüngung wohl den Radialzuwachs erhöht, jedoch gleichzeitig eine Abnahme der Rohdichte des Holzes mit sich bringt. Diese Wirkung scheint mit den Jahren nach der Düngung zuzunehmen. 
In ein und derselben Versuchsanlage und für die gleiche Behandlungsart konnte eine relative Verminderung der Rohdichte (= Infradensité : Verhältnis von Trockengewicht zu wassergesättigten Volumen) von $1,7 \%$ nach 7 Jahren festgestellt werden (Tab. 2). Zur Bestimmung wurde eine Bohrspanstichprobe verwendet, bei welcher die sich in Marknähe befindlichen Jahrringe von geringer Dichte in Überzahl vorhanden sind. An Stammstücken, an denen in der Höhe der Probennahme (ungefähr $10 \mathrm{~cm}$ vom Boden) alle Jahreszuwächse vorhanden waren, ergab sich eine Dichteverminderung von 6,3\% nach 9 Jahren (Tab. 7) und von 7,4\%, wenn bei gleichem Alter nur die drei letzten Jahrringe verwendet werden (Tab. 5).

Die Untersuchung der Dichtevariationen des Holzes (Tab. 3) zeigt, dass die Düngung vorwiegend die jährlichen Dichtemaxima verringert wodurch die Heterogenität des Holzes vermindert wird. Im übrigen konnte festgestellt werden, dass der prozentuelle Anteil an dichtem Holz an Bäumen die eine Phosphordüngung erhalten hatten signifikant geringer ist.

Unter weiteren untersuchten Qualitätsmerkmalen konnte eine nicht signifikante Erhöhung der Schwindung in der Längsrichtung des Holzes bei gedüngten Bäumen festgestellt werden. Bezüglich der Papierholz-Eigenschaften wurde in den mit Phosphor gedüngten Parzellen eine signifikant höhere Fasermenge als in den Vergleichsflächen gefunden (Tab. 17). Schliesslich zeigte sich, dass, im Gegensatz zu verschiedenen Feststellungen in der einschlägigen Literatur, die mittlere Faserlänge in den gedüngten Parzellen grösser ist ; der Unterschied ist jedoch nicht statistisch gesichert.

\section{RÉFÉRENCES BIBLIOGRAPHIQUES}

Dadswell H.E., 1963. The growth-wood property interrelationships. Proc. Special Field Inst. in Forest Biology (1960). School of Forestry N.C. State College, 1-88.

Erickson H.D. et LAMBert G.M., 1958. Effects of fertilization and thinning on chemical composition, growth, and specific gravity of young Douglas fir. For. Sci., 4, 307-315.

Guinaudeau J., Illy G., Mauge J.P. et Dumas F., 1963. Essai de fertilisation minérale sur Pin maritime à Mimizan (Landes). Ann. Ec. nation. Eaux et Forêts et Stn Rech. Expér., 20, 1.

Illy G., 1966. Recherches sur l'amélioration génétique du Pin maritime. Ann. Sci. forest., $23,4$.

KELler R., 1968. Des caractéristiques nouvelles pour l'étude des propriétés mécaniques des bois : les composantes de la densité. Ann. Sci. forest. 25, 4.

Polge H. et Illy G., 1967. Observations sur l'anisotropie du Pin maritime des Landes. Ann. Sci. forest., 24 (3), 205-231.

Polge H. et Millier C., 1967. Etude de la longueur des fibres sur échantillons prélevés à la tarière de Pressler de $5 \mathrm{~mm}$ de diamètre. Ann. Sci. forest., 24 (2), 107-119.

Posey C.E., 1964. The effect of fertilization upon wood propertie of Loblolly Pine (Pinus taeda L.). School of Forestry, North Carolina State, Raleigh, Tech. Rept, no 22.

Tomassone R., 1964. Analyse de variance d'un dispositif en blocs non orthogonal. Rev. forest. franc., $\mathrm{N}^{\circ}$ 8-9, août-septembre, 687-691.

Williams R.F. et Hamilton J.R., 1961. The effect of fertilization on four wood properties in Slash pine. J. Forest., 59 (9), 662-5.

Zobel B.J., Goggans J.F., Maki T.E. and Henson F., 1961. Some effects of fertilizers on wood properties of Loblolly Pine. Tappi, 44, 186-192. 